\title{
The Relationship Between Serum YKL-40 Levels on Admission and Stroke-Associated Pneumonia in Patients with Acute Ischemic Stroke
}

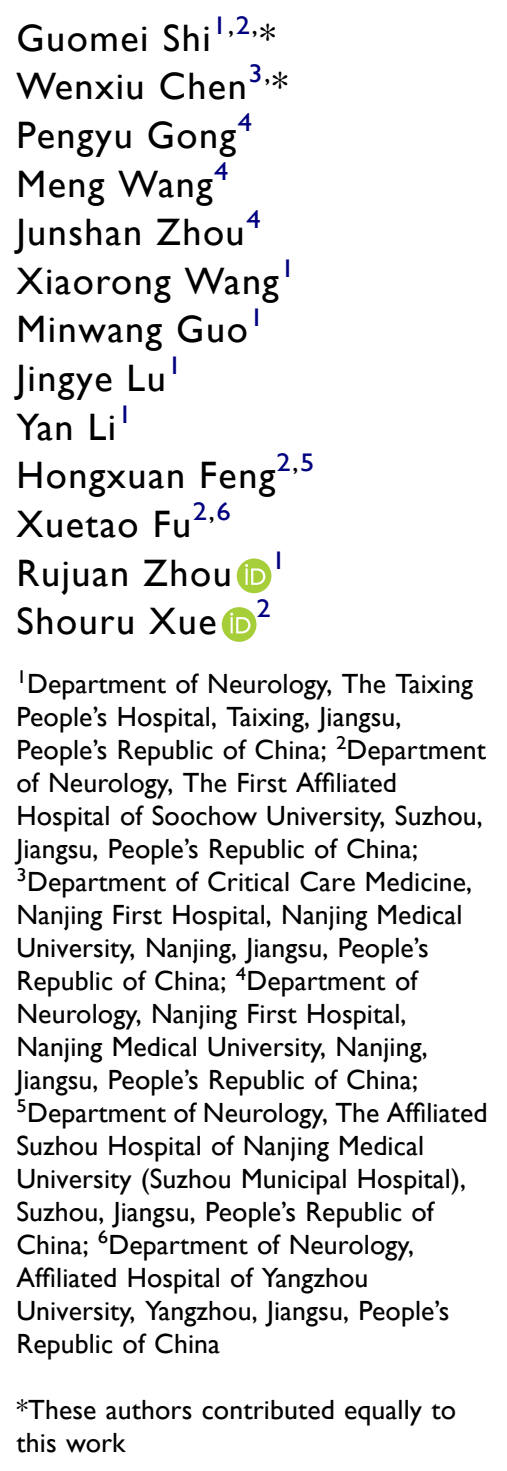

Correspondence: Rujuan Zhou; Shouru Xue

Tel +86-I395II58499; +86-I8962I33036

Email zhourujuan123@I63.com;

xueshouru@suda.edu.cn
Background: Stroke-associated pneumonia (SAP) is a standout complication after acute ischemic stroke (AIS), with a prevalence of $7-38 \%$. The aim of this prospective study was to investigate the relationship between serum YKL-40 levels at admission and SAP.

Methods: Between August 2020 and February 2021, consecutive AIS patients from two centers were enrolled prospectively. Serum YKL-40 concentrations were measured via enzyme-linked immunosorbent assay. We performed logistic regression analyses to explore the relationship between YKL-40 and SAP. Receiver operating characteristic curve was also used to assess the predictive ability of YKL-40 in predicting SAP.

Results: Ultimately, a total of 511 AIS patients were recruited. Multivariate logistic regression analysis showed that YKL-40 was independently related to SAP, whether as a continuous variable or as quartiles $(P=0.001)$. The area under curve of YKL-40 to predict SAP was 0.765 . The optimal cutoff value of YKL-40 as a predictor of SAP was determined to be $206.4 \mathrm{ng} / \mathrm{mL}$, where the sensitivity was $63.1 \%$ and the specificity was $82.0 \%$.

Conclusion: Our study demonstrated that YKL-40 might be considered as a useful biomarker to predict SAP in AIS patients.

Keywords: acute ischemic stroke, YKL-40, stroke-associated pneumonia

\section{Background}

Stroke is reported to be a serious worldwide health problem and one of the leading reasons for disability as well as mortality. ${ }^{1,2}$ Stroke-associated pneumonia (SAP) is a standout complication after acute ischemic stroke (AIS), which accounts for 7$38 \%$. $^{3,4}$ What is more, several clinical researches have verified that SAP may be linked to unfavorable prognosis and disability in AIS patients, independently. ${ }^{3-5}$ Therefore, it is crucial and clinically valuable for the neurologists to investigate the related risk factors and construct the predictive model of SAP.

Inflammatory response is getting more and more attention for its critical role in the pathological process of AIS. ${ }^{6,7}$ YKL-40, also called as chitinase-3-like-1 protein (CHI3L1) and human cartilage glycoprotein-39 (HCgp-39), is a novel inflammatory biomarker and chiefly secreted by macrophages, neutrophils and epithelial cells. ${ }^{8}$ Overexpression of YKL-40 has been observed in various inflammatory conditions including sepsis, pneumonia, asthma, diabetes, rheumatoid arthritis, and coronary artery disease. ${ }^{8,9}$ Recently, in terms of diagnosis and prognosis, YKL-40 has been proposed as a useful and meaningful biomarker for cerebrovascular diseases, such as ischemic stroke, spontaneous subarachnoid hemorrhage as well as intracerebral hemorrhage. ${ }^{10-}$ 
${ }^{12}$ Nevertheless, whether levels of YKL-40 are correlated with SAP remains unclear and wait for neurologists to explore.

The purpose of our observational research was to assess the association of serum YKL-40 levels at admission with SAP and whether serum YKL levels can predict the occurrence of SAP in AIS patients.

\section{Methods}

\section{Patient Selection}

Between August 2020 and February 2021, we recruited AIS patients hospitalized in The Taixing People's Hospital as well as Nanjing First Hospital, consecutively. All the AIS patients were admitted to the stroke centers. Eligible patients were included in the present analysis, on condition that they met all of the criteria below. The Ethics Committees of our hospitals approved this research, and all participants or their legal representatives provided signed informed consent. This research was also conducted in accordance with the Declaration of Helsinki.

\section{Inclusion and Exclusion Criteria}

Inclusion criteria: (1) admission within 48 hours of onset; (2) age $\geq 18$ years. Exclusion criteria: (1) pre-stroke pneumonia or active infection before admission; (2) patients with incomplete clinical data; (3) pre-existing dysphagia; (4) preventive antibiotic therapy; (5) The length of hospitalization is less than 7 days.

\section{Data Collection}

We collected the data of AIS patients as follows: demographic characteristics (age and gender), past medical history (hypertension, dyslipidemia, diabetes mellitus, atrial fibrillation, previous stroke, chronic obstructive pulmonary disease, smoking and drinking alcohol), clinical assessment (blood pressure, baseline National Institutes of Health Stroke Score [NIHSS], dysphagia, previous antiplatelet, previous statin, intravenous thrombolysis, tracheal intubation or ventilator, stroke subtype) as well as laboratory data (leucocyte, total cholesterol [TC], triglyceride [TG], low-density lipoprotein [LDL], highdensity lipoprotein [HDL], fasting blood glucose [FBG], hyper-sensitive C-reactive protein [Hs-CRP]). Dysphagia was identified using a bedside non-instrumented swallowing test within the first day after admission. Stroke subtype of each AIS patient was categorized in light of Trial of Org 10172 in Acute Stroke Treatment (TOAST) criteria. $^{13}$

\section{Definition of SAP}

The diagnosis of SAP was based on the modified Centers for Disease Control and Prevention criteria of hospitalacquired pneumonia, according to clinical and laboratory parameters of acute lower respiratory tract infection, and was confirmed by chest X-ray or CT. ${ }^{14,15}$ SAP was diagnosed by two trained clinicians after onset of AIS. If there is disagreement about the diagnosis of SAP, we will invite a third neurologist to make a final decision.

\section{Measurement of YKL-40}

Blood samples were collected within the first 24 hours of admission after overnight fasting. Samples were centrifuged at $1500 \times \mathrm{g}$ for $15 \mathrm{~min}$, and the isolated serum was frozen at $-80^{\circ} \mathrm{C}$ until later analysis. Serum YKL-40 levels were measured using a commercial enzyme-linked immunosorbent assay kit (Cat No. ab255719, Abcam), in line with the manufacturer's instructions, where the minimum detectable levels are $7.50 \mathrm{ng} / \mathrm{mL}$.

\section{Statistical Analysis}

Statistical analyses were conducted by R 4.0.4 (http:// www.R-project.org/). Continuous variables, which followed a normal distribution, were presented as the mean \pm standard deviation; other continuous variables, which did not follow normal distributions, were expressed as the median and interquartile range (25\% as well as $75 \%)$. Categorical variables were presented as numbers (percentages). The violin plot was used to display the distribution of YKL-40 between SAP group and non-SAP group. Differences in baseline characteristics among YKL-40 quartiles were conducted using analysis of variance or the Kruskal-Wallis test for continuous variables, and Pearson's chi-square test for categorical variables. All variables with a significant relationship at $P<0.05$ in univariate analysis entered multivariable analysis. We also conducted receiver operating characteristic (ROC) curve analysis to evaluate the predictive capability of YKL-40 in SAP and to detect the best cutoff point, where the sum of the sensitivity and specificity was the highest. The area under curve (AUC) was carried out on the basis of the ROC curve analysis. All statistical analyses were 2 -tailed, and $P<0.05$ was considered to indicate statistical significance. 


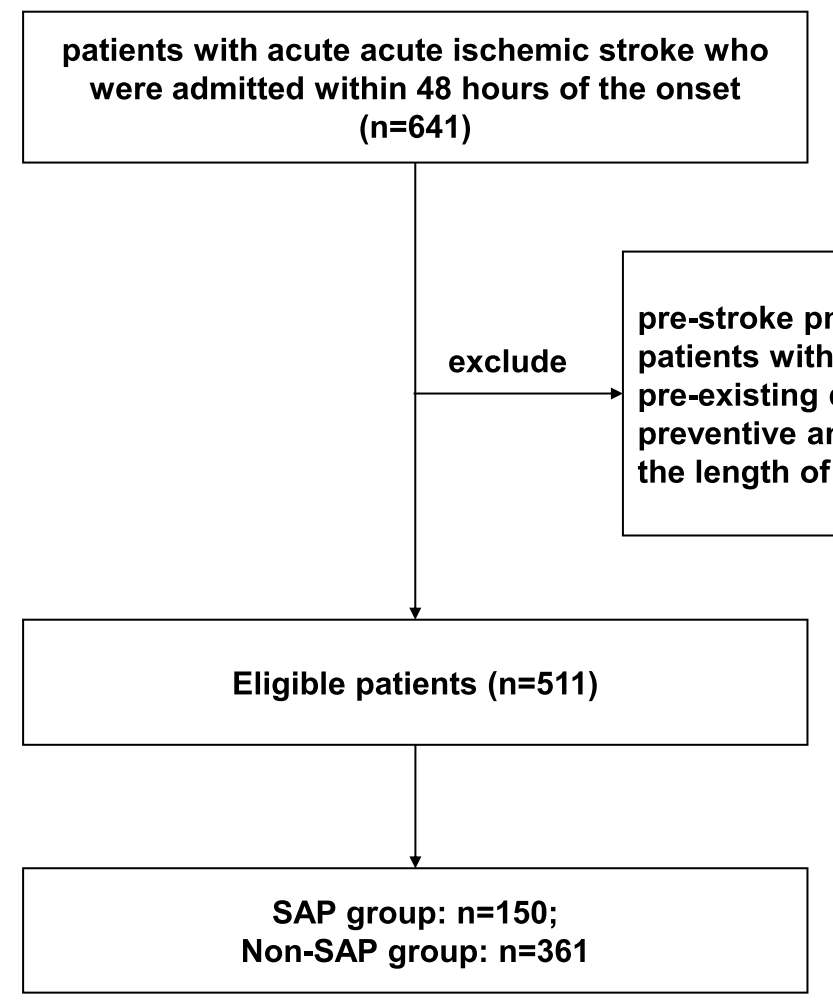

Figure I Patient flowchart.

\section{Results}

Between August 2020 and February 2021, 641 AIS patients who were admitted within 48 hours after the onset of ischemic stroke were screened in this research. One hundred and thirty AIS patients were excluded for the reasons below: pre-stroke pneumonia or active infection before admission $(\mathrm{n}=18)$; patients with incomplete clinical data $(\mathrm{n}=43)$; pre-existing dysphagia $(\mathrm{n}=7)$; preventive antibiotic therapy $(n=4)$; the length of hospitalization is less than 7 days $(\mathrm{n}=58)$. Ultimately, a total of 511 patients (328 male and 183 female) were recruited in the final analysis (Figure 1). Among the 511 patients, 150 (29.4\%) developed SAP.

The quartile levels of YKL-40 were as follows: 22.6 $\mathrm{ng} / \mathrm{mL}$ to $64.7 \mathrm{ng} / \mathrm{mL}$ (first quartile); $65.9 \mathrm{ng} / \mathrm{mL}$ to 138.6 $\mathrm{ng} / \mathrm{mL}$ (second quartile); $139.6 \mathrm{ng} / \mathrm{mL}$ to $246.5 \mathrm{ng} / \mathrm{mL}$ (third quartile); $247.4 \mathrm{ng} / \mathrm{mL}$ to $798.1 \mathrm{ng} / \mathrm{mL}$ (fourth quartile). Table 1 demonstrated the baseline characteristics of patients stratified by YKL-40 quartiles. The variables with significant differences among four groups were presented below: age $(P=0.003)$, atrial fibrillation $(P=0.011)$, coronary artery disease $(P=0.022)$, drinking alcohol $(P=0.043)$, baseline NIHSS $(P=0.001)$, dysphagia $(P=0.006)$, SAP
$(P=0.001)$, tracheal intubation or ventilator $(P=0.020)$, TOAST subtype $(P=0.005)$, leukocyte $(P=0.002)$, TG $(P=0.007)$, FBG $(P=0.001)$, as well as Hs-CRP $(P=0.001)$.

Figure 2 showed the violin plot of YKL-40 between SAP group and non-SAP group. The participants in SAP group retained elevated levels of YKL-40 than those in non-SAP group $(250.2$ [150.7, 311.5] versus 114.6 [53.4, 182.3], $P<0.001)$.

Table 2 displayed the results of logistic regression analyses for the related factors associated with SAP including YKL-40 as a continuous variable. Univariate regression analyses showed that age, atrial fibrillation, smoking, drinking alcohol, diastolic blood pressure, baseline NIHSS, dysphagia, tracheal intubation or ventilator, TOAST subtype, leucocyte counts, Hs-CRP and YKL-40 might be associated with SAP $(P<0.05)$. According to the multivariable logistic regression, which was adjusted for potential confounders mentioned above, YKL-40 (odds ratio [OR], 1.007; 95\% confidence interval [CI] 1.004 $1.009, P=0.001$ ), Hs-CRP (OR, 1.030 ; 95\% CI 1.003 $1.060, P=0.037$ ), leucocyte (OR, $1.135 ; 95 \%$ CI 1.029 $1.258, P=0.013$ ), dysphagia (OR, 4.422 ; $95 \%$ CI $2.602-$ 7.650, $P=0.001$ ), baseline NIHSS (OR, $1.146 ; 95 \% \mathrm{CI}$ $1.085-1.214, P=0.001)$ as well as diastolic blood pressure 
Table I Characteristics of Subgroups Based on the Quartiles of YKL-40

\begin{tabular}{|c|c|c|c|c|c|}
\hline Variable & $\begin{array}{l}\text { First Quartile } \\
\qquad(n=128)\end{array}$ & $\begin{array}{l}\text { Second Quartile } \\
\qquad(n=128)\end{array}$ & $\begin{array}{l}\text { Third Quartile } \\
\qquad(n=128)\end{array}$ & $\begin{array}{l}\text { Fourth Quartile } \\
\qquad(n=127)\end{array}$ & $P$ \\
\hline \multicolumn{6}{|l|}{ Demographic characteristics } \\
\hline Age (years) & $68(58,74)$ & $69(60,78)$ & $69.0(60,78)$ & $74(63,82)$ & 0.003 \\
\hline Male (\%) & $90(70.3)$ & $83(64.8)$ & $80(62.5)$ & $75(59.1)$ & 0.293 \\
\hline \multicolumn{6}{|l|}{ Past medical history (\%) } \\
\hline Hypertension & $89(69.5)$ & 91 (7I.I) & $89(69.5)$ & $88(69.3)$ & 0.989 \\
\hline Diabetes mellitus & $29(22.7)$ & $29(22.7)$ & $23(18.0)$ & $32(25.2)$ & 0.568 \\
\hline Dyslipidemia & $14(10.9)$ & $17(13.3)$ & $23(18.0)$ & $16(12.6)$ & 0.400 \\
\hline Atrial fibrillation & $19(14.8)$ & $12(9.4)$ & $25(19.5)$ & $31(24.4)$ & 0.011 \\
\hline Previous stroke & $21(16.4)$ & $24(18.9)$ & $18(14.1)$ & $28(22.0)$ & 0.383 \\
\hline Coronary artery disease & $12(9.4)$ & $15(11.8)$ & $17(13.3)$ & $28(22.5)$ & 0.022 \\
\hline COPD & $\mathrm{I}(0.8)$ & $3(2.3)$ & $7(5.5)$ & $2(1.6)$ & 0.089 \\
\hline Smoking & & & & & 0.107 \\
\hline Never & $66(51.6)$ & $74(57.8)$ & $74(57.8)$ & $80(63.0)$ & \\
\hline Ever smoking & $40(31.3)$ & $41(32.0)$ & $46(35.9)$ & $35(27.6)$ & \\
\hline Currently smoking & $22(17.2)$ & $13(10.2)$ & $8(6.3)$ & $12(9.4)$ & \\
\hline Drinking alcohol & & & & & 0.043 \\
\hline Never & $78(60.9)$ & $86(67.2)$ & $81(63.3)$ & $86(67.7)$ & \\
\hline Ever drinking alcohol & $29(22.7)$ & $29(22.7)$ & $40(31.3)$ & $33(26.0)$ & \\
\hline Currently drinking alcohol & $21(16.4)$ & $13(10.2)$ & $7(5.5)$ & $8(6.3)$ & \\
\hline \multicolumn{6}{|l|}{ Clinical assessment } \\
\hline Systolic blood pressure $(\mathrm{mmHg})$ & $155(138,166)$ & $150(140,162)$ & $147(133,160)$ & $147(134,166)$ & 0.215 \\
\hline Diastolic blood pressure $(\mathrm{mmHg})$ & $89(80,99)$ & $87(80,98)$ & $87(79,99)$ & $84(76,92)$ & 0.101 \\
\hline Baseline NIHSS & $3(2,5)$ & $4(2,6)$ & $6(3,12)$ & II $(4,15)$ & 0.001 \\
\hline Dysphagia (\%) & $32(25.0)$ & $32(25.0)$ & $38(29.7)$ & $54(42.5)$ & 0.006 \\
\hline Previous antiplatelet (\%) & $14(10.9)$ & $22(17.2)$ & $13(10.2)$ & $26(20.5)$ & 0.056 \\
\hline Previous statin (\%) & $8(6.3)$ & $19(14.8)$ & $12(9.4)$ & $19(15.0)$ & 0.072 \\
\hline Intravenous thrombolysis (\%) & $40(31.3)$ & $42(32.8)$ & $46(36.0)$ & $44(34.6)$ & 0.867 \\
\hline SAP (\%) & $13(10.2)$ & $22(17.2)$ & $37(28.9)$ & $77(60.6)$ & 0.001 \\
\hline Tracheal intubation or ventilator (\%) & $0(0.0)$ & $4(3.1)$ & $3(2.3)$ & II (8.7) & 0.020 \\
\hline TOAST subtype & & & & & 0.005 \\
\hline LAA & $53(4 I .4)$ & $61(47.7)$ & $60(46.9)$ & $66(52.0)$ & \\
\hline CE & $21(16.4)$ & $16(12.5)$ & $31(24.2)$ & $34(26.8)$ & \\
\hline SAO & $49(38.23)$ & $44(34.4)$ & $32(25.0)$ & $18(14.2)$ & \\
\hline SOE & $2(1.6)$ & $0(0.0)$ & $2(1.6)$ & $3(2.4)$ & \\
\hline SUE & $3(2.3)$ & $7(5.5)$ & $3(2.3)$ & $6(4.7)$ & \\
\hline \multicolumn{6}{|l|}{ Laboratory data } \\
\hline Leukocyte $\left(10^{\wedge 9} / \mathrm{L}\right)$ & $7.37(5.83,9.10)$ & $7.14(5.78,8.55)$ & $8.01(6.21,9.79)$ & $8.3(6.47,10.39)$ & 0.002 \\
\hline $\mathrm{TC}(\mathrm{mmol} / \mathrm{L})$ & $4.70(4.10,5.26)$ & $4.35(3.89,5.21)$ & $4.46(3.84,5.17)$ & $4.33(3.79,5.16)$ & 0.273 \\
\hline $\mathrm{TG}(\mathrm{mmol} / \mathrm{L})$ & $1.37(0.89,1.89)$ & $1.30(0.91,1.94)$ & $1.19(0.88,1.65)$ & $1.05(0.77,1.54)$ & 0.007 \\
\hline $\mathrm{HDL}(\mathrm{mmol} / \mathrm{L})$ & $1.07(0.91,1.32)$ & I.I3 (0.9I, I.32) & $\mathrm{I} .14(0.99, \mathrm{I} .40)$ & $1.18(0.99,1.40)$ & 0.093 \\
\hline LDL (mmol/L) & $2.75(2.1,3.14)$ & $2.58(2.01,3.19)$ & $2.59(2.12,3.23)$ & $2.44(1.94,3.28)$ & 0.706 \\
\hline $\mathrm{FBG}(\mathrm{mmol} / \mathrm{L})$ & $5.69(5.0 \mathrm{I}, 6.7 \mathrm{I})$ & $5.66(4.93,7.04)$ & $5.64(4.82,6.97)$ & $6.56(5.37,8.15)$ & 0.001 \\
\hline Hs-CRP (mg/L) & $2.15(0.89,5.50)$ & $2.48(1.14,6.28)$ & $4.15(1.7,9.38)$ & $6.45(3.19,13.52)$ & 0.001 \\
\hline
\end{tabular}

Abbreviations: COPD, chronic obstructive pulmonary disease; NIHSS, National Institute of Health Stroke Scale; SAP, stroke-associated pneumonia; TOAST, Trial of Org 10172 in Acute Stroke Treatment; LAA, large-artery atherosclerosis; CE, cardioembolism; SAO, small-artery occlusion; SOE, stroke of other determined etiology; SUE, stroke of undetermined etiology; TC, total cholesterol; TG, triglyceride; HDL, high-density lipoprotein; LDL, low-density lipoprotein; FBG, fasting blood glucose; Hs-CRP, hyper-sensitive C-reactive protein. 


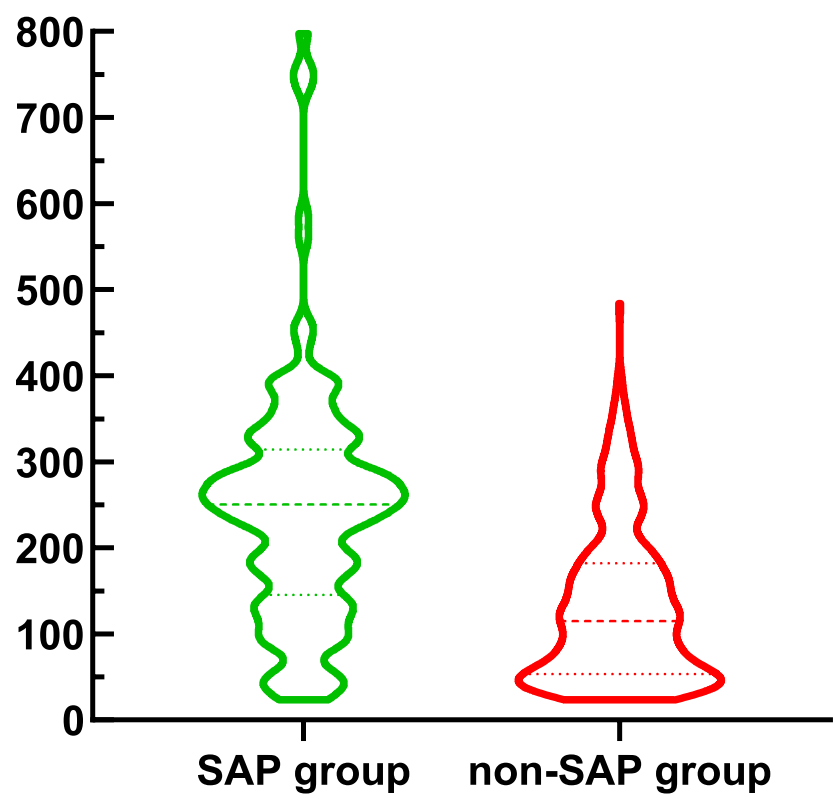

Figure 2 Violin plot in the distribution of YKL-40 between SAP group and non-SAP group.

(OR, 0.978; 95\% CI 0.957-0.998, $P=0.033$ ) were distinguished as independent related elements of SAP.

Furthermore, Table 3 showed the results of logistic regression analyses for the related factors associated with SAP including YKL-40 as quartiles. After adjusting for all potential confounders, Hs-CRP, leucocyte, dysphagia, baseline NIHSS, diastolic blood pressure and the fourth quartile of YKL-40 (first quartile used as the reference value) were identified as independent predictors for SAP. Compared with the first quartile, patients with YKL-40 levels in the fourth quartile were 5.819 times (95\% CI 2.652-13.493, $P=0.001)$ more likely to develop SAP.

To further verified the predictive ability of YKL-40 in discriminating SAP, a ROC curve analysis was conducted as depicted in Figure 3. It showed that the optimal cutoff value of YKL-40 for the diagnosis of SAP was determined to be $206.4 \mathrm{ng} / \mathrm{mL}$, which yielded a sensitivity of $63.1 \%$ and a specificity of $82.0 \%$, with the AUC of $0.765(95 \%$ CI, 0.726-0.802).

\section{Discussion}

In this hospital-based prospective observational study, we found that YKL-40 was a reliable predictor of SAP in AIS patients. The optimal cutoff value of YKL-40 to discriminate SAP was $206.4 \mathrm{ng} / \mathrm{mL}$, and its corresponding sensitivity and specificity were $63.1 \%$ and $82.0 \%$, respectively. The AUC of YKL-40 with the ability to predict SAP is 0.765. In general, a biomarker with AUC, which ranges from 0.70 to 0.90 , represents a moderate diagnostic ability. Therefore, YKL-40 may be capable of predicting SAP.

In our study, the prevalence of $29.4 \%$ for SAP was in parallel with past researches sharing the same SAP definition. ${ }^{3,4}$ Our previous study presented a much higher frequency of stroke-associated infection (65.8\%) in patients treated with endovascular therapy. ${ }^{16}$ This discrepancy may be attributed to the inclusion of different definitions about infection and higher baseline NIHSS in patients undergoing endovascular therapy. The results of our study also revealed that patients with SAP had elevated levels of Hs-CRP, leucocyte, and a higher proportion of dysphagia as well as higher baseline NIHSS, which were consistent with previous studies. ${ }^{15,17}$ Additionally, our present study found that SAP is associated with diastolic blood pressure, which has never been reported and is warranted to be investigated in future study.

During the pathogenesis and progression of brain ischemic, neuroinflammation may be extraordinarily critical. $^{6,7,18}$ Several inflammatory biomarkers were evaluated in our previous studies, ${ }^{15,16}$ suggesting that inflammatory biomarkers could be considered as prognosticators and indicators in ischemic stroke. In recent years, YKL-40, a novel biomarker of inflammation, has attracted attention as a valuable tool to predict diseases that are characterized by inflammation, fibrosis and tissue remodeling. ${ }^{8,9}$ In pulmonary diseases such as sepsis, community-acquired pneumonia as well as chronic obstructive pulmonary disease, YKL-40 was found to be correlated with disease severity. ${ }^{19-}$ ${ }^{21}$ Currently, YKL-40 is not only associated with pulmonary diseases, but also considered to be an independent biomarker of cardiovascular and cerebrovascular diseases, such as carotid artery atherosclerosis, myocardial infarction, ischemic stroke, spontaneous subarachnoid hemorrhage and intracerebral hemorrhage. ${ }^{10-12,22}$ Park et al reported that YKL-40 levels might be associated with stroke severity, infarct volume and clinical outcome of 105 Korean AIS patients. ${ }^{10}$ However, this is a small-size study without prospective information. Another clinical study in China evaluated YKL-40 in 141 stroke patients and found the patients with elevated serum YKL-40 levels might develop unfavorable clinical outcome. ${ }^{23}$ Nevertheless, the finding was limited to patients with large-artery atherosclerosis stroke. It is unknown whether YKL-40 is also associated with SAP in AIS patients. To our knowledge, it was the first time that the possible correlation between YKL-40 and SAP in Chinese AIS patients was prospectively assessed, with a relatively large sample size. 
Table 2 Logistic Regression Analyses for the Related Factors Associated with SAP Including YKL-40 as a Continuous Variable

\begin{tabular}{|c|c|c|c|c|}
\hline Variable & $\begin{array}{l}\text { Unadjusted OR (95\% } \\
\mathrm{CI})\end{array}$ & $\mathbf{P}$ & Adjusted OR (95\% Cl) & $P$ \\
\hline \multicolumn{5}{|l|}{ Demographic characteristics } \\
\hline Age & $1.032(1.016-1.049)$ & 0.001 & $1.004(0.979-1.030)$ & 0.755 \\
\hline Male & $0.763(0.516-1.133)$ & 0.178 & & \\
\hline \multicolumn{5}{|l|}{ Past medical history } \\
\hline Hypertension & $0.87 \mid(0.579-|.32|)$ & 0.512 & & \\
\hline Diabetes mellitus & $0.897(0.557-1.418)$ & 0.648 & & \\
\hline Dyslipidemia & $0.89 \mid(0.496-1.545)$ & 0.690 & & \\
\hline Atrial fibrillation & $2.608(1.620-4.193)$ & 0.001 & $0.694(0.263-1.809)$ & 0.457 \\
\hline Coronary heart disease & $1.448(0.848-2.429)$ & 0.167 & & \\
\hline Chronic obstructive pulmonary disease & $2.128(0.674-6.512)$ & 0.181 & & \\
\hline Previous stroke & $1.486(0.915-2.385)$ & 0.104 & & \\
\hline \multicolumn{5}{|l|}{ Smoking } \\
\hline Never & Reference & & Reference & \\
\hline Ever smoking & $0.856(0.56 \mathrm{I}-\mathrm{I} .296)$ & 0.467 & $1.289(0.59 \mid-2.795)$ & 0.521 \\
\hline Currently smoking & $0.305(0.122-0.659)$ & 0.005 & $0.894(0.171-3.965)$ & 0.888 \\
\hline \multicolumn{5}{|l|}{ Drinking alcohol } \\
\hline Never & Reference & & Reference & \\
\hline Ever drinking alcohol & $0.879(0.56|-| .362)$ & 0.570 & $0.897(0.409-1.954)$ & 0.785 \\
\hline Currently drinking alcohol & $0.300(0.112-0.677)$ & 0.008 & $0.349(0.062-1.897)$ & 0.222 \\
\hline \multicolumn{5}{|l|}{ Clinical assessment } \\
\hline Systolic blood pressure & 1.001 (0.999-1.003) & 0.342 & & \\
\hline Diastolic blood pressure & $0.969(0.954-0.983)$ & 0.001 & $0.978(0.957-0.998)$ & 0.033 \\
\hline Baseline NIHSS & $1.211(1.167-1.260)$ & 0.001 & $1.146(1.085-1.214)$ & 0.001 \\
\hline Dysphagia & $4.000(2.667-6.032)$ & 0.001 & $4.422(2.602-7.650)$ & 0.001 \\
\hline Previous antiplatelet & $1.088(0.629-1.834)$ & 0.756 & & \\
\hline Previous statin & $0.917(0.485-1.657)$ & 0.780 & & \\
\hline Intravenous thrombolysis & $1.225(0.820-1.822)$ & 0.318 & & \\
\hline Tracheal intubation or ventilator & 9.281 (3.264-33.188) & 0.001 & $0.408(0.082-2.339)$ & 0.288 \\
\hline \multicolumn{5}{|l|}{ TOAST subtype } \\
\hline LAA & Reference & & Reference & \\
\hline CE & $2.379(1.480-3.839)$ & 0.001 & $2.072(0.8 \mid 4-5.323)$ & 0.127 \\
\hline SAO & $0.210(0.104-0.389)$ & 0.001 & $0.812(0.355-1.786)$ & 0.611 \\
\hline SOE & $0.915(0.129-4.353)$ & 0.917 & $0.439(0.035-3.401)$ & 0.469 \\
\hline SUE & $2.542(0.985-6.653)$ & 0.052 & $2.249(0.550-9.306)$ & 0.259 \\
\hline \multicolumn{5}{|l|}{ Laboratory data } \\
\hline Leucocyte & $1.208(1.128-1.299)$ & 0.001 & 1.135 (I.029-1.258) & 0.013 \\
\hline $\mathrm{TC}$ & $0.847(0.706-1.009)$ & 0.068 & & \\
\hline TG & $0.87 \mid(0.719-1.006)$ & 0.130 & & \\
\hline HDL & $1.422(0.803-2.505)$ & 0.223 & & \\
\hline LDL & $0.859(0.689-0.987)$ & 0.169 & & \\
\hline FBG & $0.999(0.979-1.009)$ & 0.890 & & \\
\hline $\mathrm{Hs}$-CRP & $1.078(1.054-1.105)$ & 0.001 & $1.030(1.003-1.060)$ & 0.037 \\
\hline YKL-40 & $1.010(1.008-1.012)$ & 0.001 & $1.007(1.004-1.009)$ & 0.001 \\
\hline
\end{tabular}

Abbreviations: SAP, stroke-associated pneumonia; NIHSS, National Institute of Health Stroke Scale; TOAST, Trial of Org I0I72 in Acute Stroke Treatment; LAA, large-artery atherosclerosis; CE, cardioembolism; SAO, small-artery occlusion; SOE, stroke of other determined etiology; SUE, stroke of undetermined etiology; TC, total cholesterol; TG, triglyceride; HDL, high-density lipoprotein; LDL, low-density lipoprotein; FBG, fasting blood glucose; Hs-CRP, hyper-sensitive C-reactive protein. 
Table 3 Multivariable Logistic Analyses for the Related Factors Associated with SAP Including YKL-40 as Quartiles

\begin{tabular}{|c|c|c|}
\hline Variable & $\begin{array}{c}\text { Adjusted OR } \\
(95 \% \mathrm{Cl})\end{array}$ & $P$ \\
\hline Age & $1.004(0.980-1.029)$ & 0.733 \\
\hline Atrial fibrillation & $0.738(0.282-1.903)$ & 0.532 \\
\hline \multicolumn{3}{|l|}{ Smoking } \\
\hline Never & Reference & Reference \\
\hline Ever smoking & $1.322(0.608-2.859)$ & 0.478 \\
\hline Currently smoking & $0.954(0.188-4.214)$ & 0.952 \\
\hline \multicolumn{3}{|l|}{ Drinking alcohol } \\
\hline Never & Reference & Reference \\
\hline Ever drinking alcohol & $0.876(0.399-1.908)$ & 0.740 \\
\hline Currently drinking alcohol & $0.448(0.085-2.319)$ & 0.335 \\
\hline Diastolic blood pressure & $0.976(0.955-0.995)$ & 0.017 \\
\hline Baseline NIHSS & 1.149 (1.088-1.217) & 0.001 \\
\hline Dysphagia & $4.253(2.516-7.308)$ & 0.001 \\
\hline Tracheal intubation or ventilator & $0.419(0.087-2.305)$ & 0.293 \\
\hline \multicolumn{3}{|l|}{ TOAST subtype } \\
\hline LAA & Reference & Reference \\
\hline CE & $2.01 \mathrm{I}(0.802-5.08 \mathrm{I})$ & 0.136 \\
\hline SAO & $0.704(0.307-1.544)$ & 0.391 \\
\hline SOE & $0.416(0.035-3.230)$ & 0.437 \\
\hline SUE & $1.976(0.478-8.269)$ & 0.348 \\
\hline Leucocyte & $1.140(1.034-1.261)$ & 0.010 \\
\hline $\mathrm{Hs}-\mathrm{CRP}$ & $1.029(1.003-1.058)$ & 0.033 \\
\hline \multicolumn{3}{|l|}{ YKL-40 } \\
\hline First quartile & Reference & Reference \\
\hline Second quartile & I.574 (0.67I-3.8I2) & 0.303 \\
\hline Third quartile & $\mathrm{I} .620(0.70 \mathrm{I}-3.866)$ & 0.265 \\
\hline Fourth quartile & $5.819(2.652-13.493)$ & 0.001 \\
\hline
\end{tabular}

Abbreviations: SAP, stroke-associated pneumonia; NIHSS, National Institute of Health Stroke Scale; TOAST, Trial of Org 10172 in Acute Stroke Treatment; LAA, large-artery atherosclerosis; $\mathrm{CE}$, cardioembolism; SAO, small-artery occlusion; SOE, stroke of other determined etiology; SUE, stroke of undetermined etiology; Hs-CRP, hyper-sensitive C-reactive protein.

The exact pathophysiological mechanisms responsible for elevated YKL-40 concentrations with SAP are unclear. First of all, it is generally accepted that YKL- 40 is a glycoprotein produced by neutrophils as well as macrophages in response to inflammation or infection ${ }^{9,21}$ and thus provides knowledge regarding the risk of pneumonia. Moreover, it is well-known that inflammatory process is involved in the pathological development of ischemic stroke, from pre-stroke arteriosclerosis to post-stroke brain damage, ${ }^{6,7,18}$ among which YKL-40 is one of the

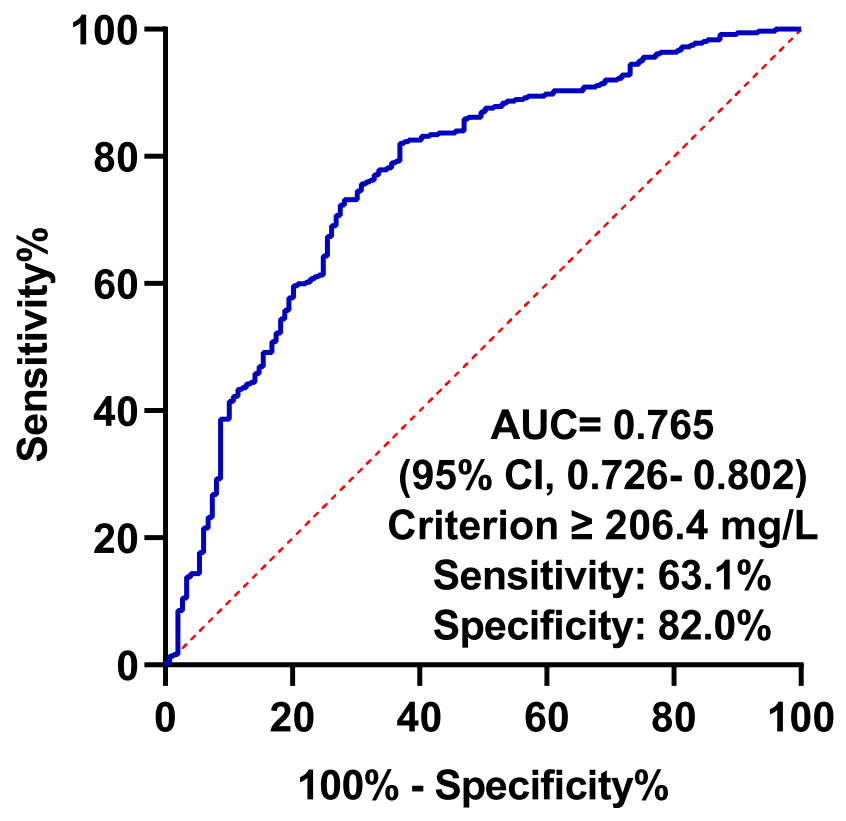

Figure 3 ROC curve for values of YKL-40 to predict SAP.

most recognized mediators. YKL-40, on one hand, can activate endothelial cells to express intercellular adhesion molecule-1 as well as vascular adhesion molecule-1, which would further impair the vascular endothelial cells, promote vascular smooth muscle cells activation, and eventually exacerbate the development of atherosclerosis. $^{24}$ On the other hand, the expression of YKL-40 could be activated by pro-inflammatory cytokines, such as tumor necrosis factor- $\alpha$, interleukin (IL)$1 \beta$ and IL-6, which are synthesized from microglia and macrophages in penumbra after ischemic stroke. ${ }^{25,26}$ These may be significant contributors to YKL-40 with SAP. Furthermore, Kim et al have shown that treatment with anti-CHI3L1 antibody could vitiate Th2 cytokine production as well as airway inflammation in respiratory syncytial virus infected animal model, suggesting an immunologic property of YKL-40. ${ }^{27}$ By contrast, in another pre-clinical animal model, Chi3L1 knockout deteriorated ischemia/reperfusion damage. ${ }^{28}$ It is unclear whether the management of serum YKL-40 levels within the appropriate range is beneficial or harmful in AIS, which deserved to be further investigated in the future.

Nevertheless, in our observational clinical research, there still exist several limitations that should be taken into account in further investigations. Firstly, the sample size of this observational investigation was not big, relatively. Secondly, several biomarkers, which may be related with SAP owing to the previous studies, have not been 
examined in this study, such as serum iron, lipopolysaccharide binding protein and sCD14. ${ }^{29,30}$ Thirdly, some studies have shown the association of immunosuppression and other unstable conditions such as renal failure, hepatic failure and cancer with SAP. ${ }^{31,32}$ However, these factors were not measured in our study protocol, and we did not exclude the effects of these factors on SAP. Also, we cannot totally exclude the underlying effect of other undetected conditions such as stress biomarkers, genetic predisposition on YKL-40 and COVID-19 status. Fourthly, we only investigated the baseline levels of YKL-40, and did not study the dynamic changes of YKL-40 levels. Serial measurements of YKL-40 may provide more objective and comprehensive information to verify the predictive value of YKL-40. Moreover, we did not recruit an external verification cohort to confirm the conclusions of our study. In addition, according to the strict exclusion criteria and assessment, quite a few AIS patients were excluded, which might result in bias. Because of all the shortcomings mentioned above, we plan to carry out more well-designed prospective observational clinical trials with a larger sample size about SAP in the future.

\section{Conclusion}

In summary, serum YKL-40 levels may be a valuable noninvasive diagnostic biomarker for the occurrence of SAP in AIS patients, in light of the results as well as the conclusion of our observational research. It is useful for the neurologists to utilize serum levels of YKL-40 in the management of SAP in the clinical practice.

\section{Data Sharing Statement}

The data that support the findings of this study are available from Rujuan Zhou upon reasonable request.

\section{Ethics Approval and Consent to Participate}

Informed consent was obtained from participants or legal representatives, and the protocol was approved by the Ethical Committee of The Taixing People's Hospital and Nanjing First Hospital. The protocol was also conducted in accordance with the Declaration of Helsinki.

\section{Acknowledgments}

We express our gratitude to all the patients who participated in this study.

\section{Author Contributions}

All authors made a significant contribution to the work reported, whether that is in the conception, study design, execution, acquisition of data, analysis, and interpretation, or in all these areas; took part in drafting, revising, or critically reviewing the article; gave final approval of the version to be published; have agreed on the journal to which the article has been submitted; and agree to be accountable for all aspects of the work.

\section{Funding}

This work was supported by the Key Program of Kangda College of Nanjing Medical University Science and Technique Development Foundation Project (KD2020KYJJZD019) and the Young Scientific and Technological Talents Promotion Project of Jiangsu Association for Science and Technology (TJ2021019).

\section{Disclosure}

The authors report no conflicts of interest in this work.

\section{References}

1. Feigin V, Forouzanfar M, Krishnamurthi R, et al. Global and regional burden of stroke during 1990-2010: findings from the Global Burden of Disease Study 2010. Lancet. 2014;383(9913):245-254. doi:10.1016/s0140-6736(13)61953-4

2. Wang W, Jiang B, Sun H, et al. Prevalence, incidence, and mortality of stroke in China: results from a nationwide population-based survey of 480,687 adults. Circulation. 2017;135(8):759-771. doi:10.1161/ circulationaha.116.025250

3. Hotter B, Hoffmann S, Ulm L, et al. External validation of five scores to predict stroke-associated pneumonia and the role of selected blood biomarkers. Stroke. 2021;52(1):325-330. doi:10.1161/strokeaha.1 20.031884

4. Nam K, Kim T, Lee J, et al. High neutrophil-to-lymphocyte ratio predicts stroke-associated pneumonia. Stroke. 2018;49(8):1886-1892. doi:10.1161/strokeaha.118.021228

5. Ji R, Shen H, Pan Y, et al. Novel risk score to predict pneumonia after acute ischemic stroke. Stroke. 2013;44(5):1303-1309. doi:10.1161/ strokeaha.111.000598

6. Gong P, Liu Y, Gong Y, et al. The association of neutrophil to lymphocyte ratio, platelet to lymphocyte ratio, and lymphocyte to monocyte ratio with post-thrombolysis early neurological outcomes in patients with acute ischemic stroke. J Neuroinflammation. 2021;18 (1):51. doi:10.1186/s12974-021-02090-6

7. Lambertsen KL, Finsen B, Clausen BH. Post-stroke inflammationtarget or tool for therapy? Acta Neuropathol. 2018;137(5):693-714. doi:10.1007/s00401-018-1930-Z

8. Zhao T, Su Z, Li Y, Zhang X, You Q. Chitinase-3 like-protein-1 function and its role in diseases. Signal Transduct Target Ther. 2020;5(1):201. doi:10.1038/s41392-020-00303-7

9. Yeo I, Lee C, Han S, Yun J, Hong J. Roles of chitinase 3-like 1 in the development of cancer, neurodegenerative diseases, and inflammatory diseases. Pharmacol Ther. 2019;203:107394. doi:10.1016/j. pharmthera.2019.107394 
10. Park H, Jun C, Jeon S, et al. Serum YKL-40 levels correlate with infarct volume, stroke severity, and functional outcome in acute ischemic stroke patients. PLoS One. 2012;7(12):e51722. doi:10.1371/journal.pone.0051722

11. Lad S, Hegen H, Gupta G, Deisenhammer F, Steinberg G. Proteomic biomarker discovery in cerebrospinal fluid for cerebral vasospasm following subarachnoid hemorrhage. J Stroke Cerebrovasc Dis. 2012;21(1):30-41. doi:10.1016/j.jstrokecerebrovasdis.2010.04.004

12. Jiang Y, Zhang G, Wang Z, Yang H. Serum YKL-40 levels as a prognostic factor in patients with intracerebral hemorrhage. Clin Biochem. 2014;47(18):302-306. doi:10.1016/j. clinbiochem.2014.09.015

13. Adams HP, Bendixen BH, Kappelle LJ, et al. Classification of subtype of acute ischemic stroke. Definitions for use in a multicenter clinical trial. TOAST. Trial of Org 10172 in Acute Stroke Treatment. Stroke. 1993;24(1):35-41. doi:10.1161/01.str.24.1.35

14. Smith CJ, Kishore AK, Vail A, et al. Diagnosis of stroke-associated pneumonia: recommendations from the pneumonia in stroke consensus group. Stroke. 2015;46(8):2335-2340. doi:10.1161/ strokeaha.115.009617

15. Shi G, Li M, Zhou R, et al. Procalcitonin related to stroke-associated pneumonia and clinical outcomes of acute ischemic stroke after IV rt-PA treatment. Cell Mol Neurobiol. 2021;1-9. doi:10.1007/s10571020-01031-w

16. Deng Q, Gong P, Chen X, et al. Admission blood cell counts are predictive of stroke-associated infection in acute ischemic stroke patients treated with endovascular therapy. Neurol Sci. 2021;42 (6):2397-2409. doi:10.1007/s10072-020-04827-2

17. Wang Q, Liu Y, Han L, et al. Risk factors for acute stroke-associated pneumonia and prediction of neutrophil-to-lymphocyte ratios. $\mathrm{Am}$ J Emerg Med. 2021;41:55-59. doi:10.1016/j.ajem.2020.12.036

18. Shi K, Tian DC, Li ZG, Ducruet AF, Lawton MT, Shi FD. Global brain inflammation in stroke. Lancet Neurol. 2019;18 (11):1058-1066. doi:10.1016/s1474-4422(19)30078-x

19. Tong X, Wang D, Liu S, et al. The YKL-40 protein is a potential biomarker for COPD: a meta-analysis and systematic review. Int J Chron Obstruct Pulmon Dis. 2018;13:409-418. doi:10.2147/ copd.s152655

20. Kjaergaard A, Helby J, Johansen J, Nordestgaard B, Bojesen S. Elevated plasma YKL-40 and risk of infectious disease: a prospective study of 94665 individuals from the general population. Clin Microbiol Infect. 2020;26(10):1411.e1411-1411. e1419. doi:10.1016/j.cmi.2020.01.010

21. Spoorenberg S, Vestjens S, Rijkers G, et al. YKL-40, CCL18 and SP-D predict mortality in patients hospitalized with community-acquired pneumonia. Respirology. 2017;22(3):542-550. doi:10.1111/resp. 12924
22. Pala S, Sari M, Kahveci G, Alizade E, Arslantas U, Uslu A. Plasma YKL-40 elevation on admission and follow-up is associated with diastolic dysfunction and mortality in patients with acute myocardial infarction. Cardiol Res Pract. 2018;2018:8701851. doi:10.1155/ 2018/8701851

23. Chen X, Li Q, Huang W, et al. Serum YKL-40, a prognostic marker in patients with large-artery atherosclerotic stroke. Acta Neurol Scand. 2017;136(2):97-102. doi:10.1111/ane.12688

24. Yasuda T, Kaneto H, Katakami N, et al. YKL-40, a new biomarker of endothelial dysfunction, is independently associated with albuminuria in type 2 diabetic patients. Diabetes Res Clin Pract. 2011;91(2):e50e52. doi:10.1016/j.diabres.2010.11.015

25. Clausen B, Lambertsen K, Babcock A, Holm T, Dagnaes-Hansen F, Finsen B. Interleukin-1beta and tumor necrosis factor-alpha are expressed by different subsets of microglia and macrophages after ischemic stroke in mice. $J$ Neuroinflammation. 2008;5:46. doi:10.1186/1742-2094-5-46

26. Downes C, Crack P. Neural injury following stroke: are Toll-like receptors the link between the immune system and the CNS? $\mathrm{Br}$ $J \quad$ Pharmacol. 2010;160(8):1872-1888. doi:10.1111/j.14765381.2010.00864.x

27. Kim MJ, Shim DH, Cha HR, et al. Chitinase 3-like 1 protein plays a critical role in respiratory syncytial virus-induced airway inflammation. Allergy. 2018;74(4):685-697. doi:10.1111/all.13661

28. Im JH, Yeo IJ, Park PH, et al. Deletion of Chitinase-3-like 1 accelerates stroke development through enhancement of Neuroinflammation by STAT6-dependent M2 microglial inactivation in Chitinase-3-like 1 knockout mice. Exp Neurol. 2020;323:113082. doi:10.1016/j.expneurol.2019.113082

29. Li J, Feng L, Huang Q, Ren W. An L-shaped relationship between serum iron and stroke-associated pneumonia. Clin Interv Aging. 2021;16:505-511. doi:10.2147/cia.s301480

30. Gradek-Kwinta E, Czyzycki M, Lopatkiewicz A, Klimiec-Moskal E, Slowik A, Dziedzic T. Lipopolysaccharide binding protein and SCD14 as risk markers of stroke-associated pneumonia. J Neuroimmunol. 2021;354:577532. doi:10.1016/j. jneuroim.2021.577532

31. Hoffmann S, Harms H, Ulm L, et al. Stroke-induced immunodepression and dysphagia independently predict stroke-associated pneumonia - the PREDICT study. J Cereb Blood Flow Metab. 2017;37 (12):3671-3682. doi:10.1177/0271678x16671964

32. Faura J, Bustamante A, Miró-Mur F, Montaner J. Stroke-induced immunosuppression: implications for the prevention and prediction of post-stroke infections. J Neuroinflammation. 2021;18(1):127. doi:10.1186/s12974-021-02177-0
Journal of Inflammation Research

\section{Publish your work in this journal}

The Journal of Inflammation Research is an international, peerreviewed open-access journal that welcomes laboratory and clinica findings on the molecular basis, cell biology and pharmacology of inflammation including original research, reviews, symposium reports, hypothesis formation and commentaries on: acute/chronic inflammation; mediators of inflammation; cellular processes; molecular mechanisms; pharmacology and novel anti-inflammatory drugs; clinical conditions involving inflammation. The manuscript management system is completely online and includes a very quick and fair peerreview system. Visit http://www.dovepress.com/testimonials.php to read real quotes from published authors. 